\title{
Measuring Three-dimensional Strain Distribution of Knee Joint Cartilage under Compressive Load
}

\author{
Maho Itakura $^{1^{*}}$, Yuelin Zhang ${ }^{2 *}$, Kyohei Takeo ${ }^{1 *}$ and Satoru Yoneyama ${ }^{1^{*}}$ \\ ${ }^{1}$ Department of Mechanical Engineering, Aoyama Gakuin University, 5-10-1 Fuchinobe, Chuo-ku, Sagamihara-shi, Kanagawa, \\ Japan \\ ${ }^{2}$ Department of Engineering and Applied Sciences, Sophia University, 7-1 kioicho, Chiyoda-ku, Tokyo, Japan \\ *c5619112@aoyama.jp
}

Introduction. Articular cartilage is a connective tissue of joints, plays a role in load transfer, impact absorption and lubrication, and so is an important tissue to facilitate daily activities. Population of elderly people has been increasing all over the world due to progress in medical technology and the prevalence of advanced medical treatment. Thus, the number of cases of osteoarthritis (OA), a disease of articular cartilage, are rapidly increasing. OA develops when aging articular cartilage is worn down and damaged. To diagnose the disease at an early stage, a non-contact diagnostic method is required.

In this research, the objective is to establish a lessinvasive method to visualize the cartilage deformation under compressive load by using Digital Volume Correlation $^{(1)}$.

Compression test and micro-CT scanning. The sample used was a distal end of the femur, $50 \mathrm{~mm}$ long sample including the suboccipital bone and the articular cartilage was taken out from the femur. The compression experiment was performed using a compression device made with acrylic (Fig.1(a)), and the image acquisition was performed using a micro-CT scanner (inspeXio SMX-100CT, SHIMAZU). The micro-CT scanning was conducted at $100 \mathrm{kV}$ and $150 \mathrm{uA}$, all of which were imported as 16bit images with a size of $512 \times 512$ pixels. The resolution of the images were $0.181 \times 0.181 \times 0.049 \mathrm{~mm}^{3} /$ voxel. The captured images are shown in Fig.1(b) and (c).

Displacement and strain distributions. Displacement and strain distributions in threedimension are calculated using the acquired volume images of unloaded and loaded conditions. The analysis area (Fig.1 (b)) is $40 \times 40 \times 25 \mathrm{~mm}^{3}$ which the deformation of the cartilage can be confirmed on the images, the arrows indicate the compression direction ( $z$ direction). The subset size used for the analysis is $11 \times 11 \times 11$ pixels to $71 \times 71 \times 71$ pixels and the gauge length when calculating the strain is $21 \times$ $21 \times 21$ pixels. The calculation step is 2 pixels in each of the $x, y$ and $z$ direction.

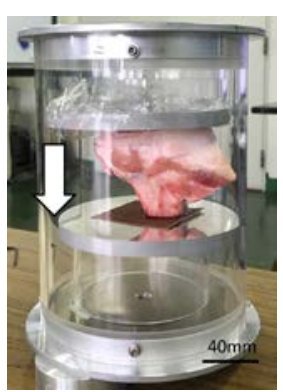

(a) Compressor

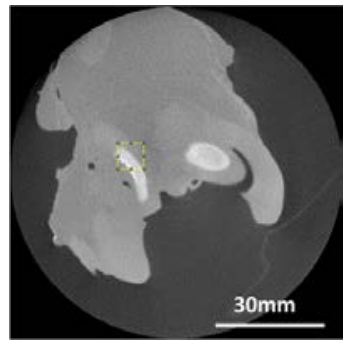

(b) before compression.

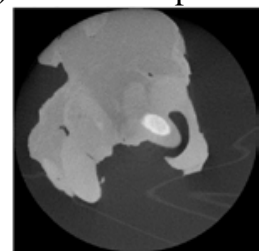

(c) after compression.

Fig. 1 Compression test and micro-CT scanning.

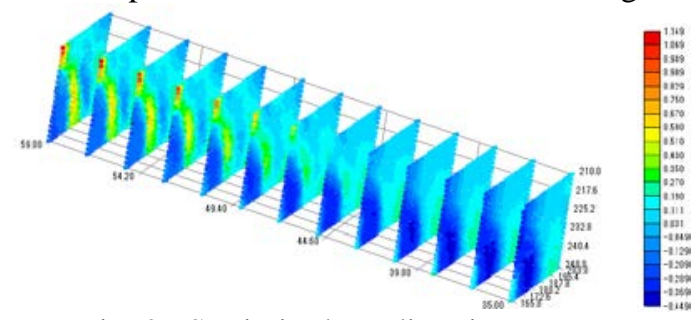

Fig. 2 Strain in the $x$ direction.

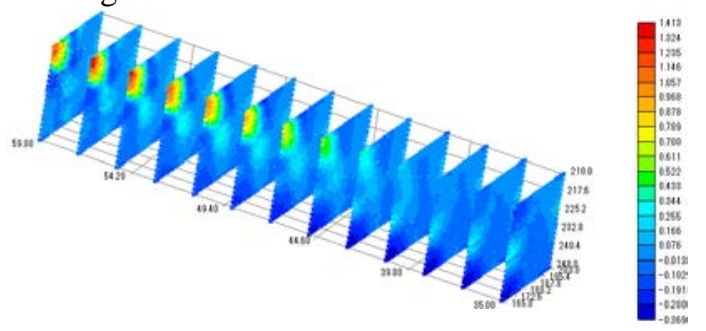

Fig. 3 Strain in the $y$ direction.

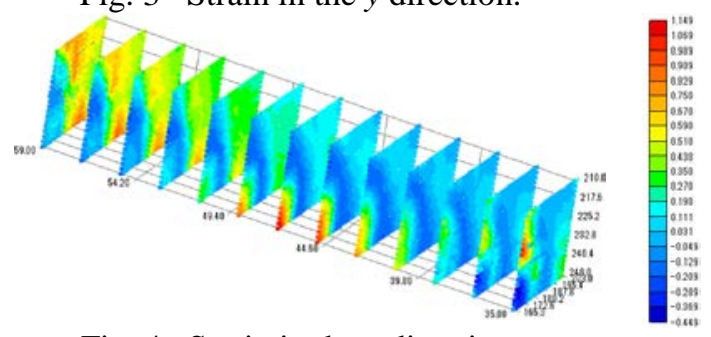

Fig. 4 Strain in the $z$ direction.

(1) Bay, B. K., Smith, T. S., Fyhrie, D. P. and M. Saad, Digital Volume Correlation: Three-dimensional Strain Mapping Using X-ray Tomography, (1999), 39, Experimental Mechanics 